\title{
Expert to present test results of hypochlorous antimicrobial irrigation at IDS Cologne
}

Professor Fadi Jarad, Honorary Consultant in Restorative Dentistry at Liverpool Dental School, will be presenting the results of his research into the pioneering use of hypochlorous acid for intracanal irrigation at the International Dental Show (IDS) trade fair in Cologne, Germany on 14 March 2019.

This work demonstrates the elimination of the common bacteria found in endodontic infection.

Professor Jarad has been working with leading specialists CleanCert, who have invested significantly in the research and development of the technology over the last three years and have launched the product as OraWize+ for intended use in endodontics, perio and implantology.

Professor Jarad said: 'My research has found that the use of OraWize+ as an irrigant has safety benefits over the tried and tested products and enables safe yet effective antibacterial irrigation.
'This is a very exciting development for the industry because it enables clinicians to utilise highly effective antibacterial irrigants without the inherent risks found with existing products.

'Hypochlorous acid is naturally occurring and this level of biocompatibility means it is harmless to patients' own cells while being lethal to bacteria. It is also environmentally friendly and extremely effective not just in eliminating dental pathogens but also safely removing the excessive biofilms that can proliferate in the treatment areas.'

Safe Endodontics with OraWize+ will take place on 14

March 2019 at 12.30 pm at 'Speakers Corner' within IDS Cologne.

CleanCert is exhibiting at Stand C068 Hall 2.2 in BDIA/UK Pavillion.

More information is available by emailing sales@ cleancert.co.uk or visiting www.cleancert.co.uk.

\section{The first of its kind}

The needs of edentulous patients can be highly variable, and finding the right treatment for each and every one can be easier said than done.

Why not expand your repertoire and help to treat more patients by choosing the Trefoil system from Nobel Biocare?

A fast and efficient new innovation, the system is unique in that it utilises the first ever pre-manufactured titanium bar capable of achieving passive fit. This, supported by three implants, creates a system that allows more patients to experience fixed and definitive teeth on the day of implant surgery.

More information is available by calling 02087563300 or visiting www.nobelbiocare.com/trefoil.

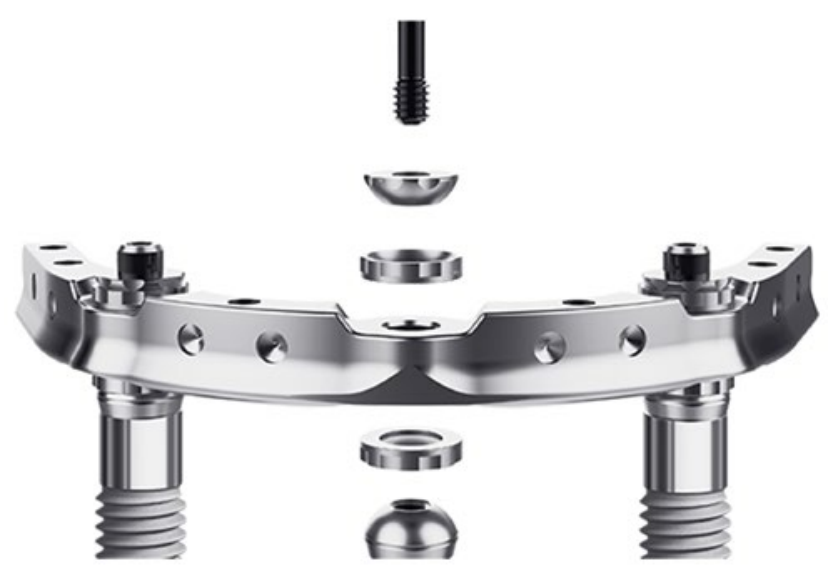

\section{Add value to your practice}

One of the three most important factors that patients value when choosing a dental practice is the use of advanced technology. So practices need to make patients aware of the modern equipment, technology and materials that are available to them, not just within the practice but also from the dental laboratory.

A custom developed, high performance polymer called Ultaire AKP is now available in the UK.

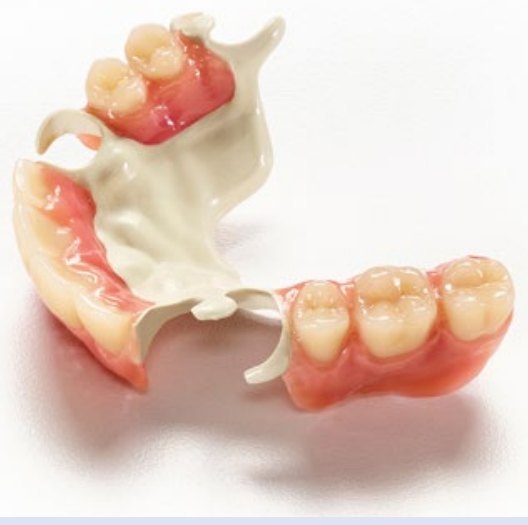

Created by Solvay Dental 360, this innovative material is ideal for the fabrication of removable partial denture (RPD) frames and offers patients a lightweight, comfortable and biocompatible alternative to metal.

Ultaire AKP has bone-like properties that many patients prefer and it provides both the elasticity and flexural strength to withstand loading.

These frames are tooth supported, retentive and stable and offer a superior overall fit that may even limit future bone loss.

By presenting the high-tech option of Ultaire AKP, practices can add a valuable distinction to treatment recommendations and patient satisfaction, said Solvay Dental 360.

More information about Solvay Dental 360, Ultaire and Dentivera milling discs is available by visiting www.solvaydental360.com. 